\title{
Lazy leucocyte syndrome-disorder of the granulocyte membrane?
}

\author{
P. H. PINKERTON, JEAN B. ROBINSON, AND J. S. SENN \\ From the Departments of Laboratory Haematology and Medicine, Sunnybrook Medical Centre and \\ Departments of Pathology and Medicine, University of Toronto, Toronto, Ontario, Canada
}

SUMMARY An adult with long-standing neutropenia had the functional granulocyte abnormalities typical of the lazy leucocyte syndrome. Scanning electron microscopy of the patient's neutrophils showed alteration in the surface configuration of the cell with coarsening of the normal fine ruffles and the appearance of knob-like projections. Similar functional and anatomical changes were induced in normal neutrophils by treatment with vinblastine. The lazy leucocyte syndrome may be a consequence of altered membrane microfilamentous protein structure or function, and undue rigidity of the affected neutrophils may explain the clinicopathological features of the disease.

Neutrophil microbicidal activity consists of a complex series of functions including migration, phagocytosis of organisms, production of bactericidal substances and their release into the phagosome, and digestion of the ingested material. A variety of congenital and acquired disorders of these functions has been described (Baehner, 1974; Gallin and Wolff, 1975; Miller, 1975; Quie, 1975), which may lead to an undue susceptibility to infection. One of the earliest congenital abnormalities of neutrophil function to be reported was of defective mobility, both random and directed, associated with peripheral neutropenia and normal numbers of mature neutrophils in the bone marrow. The abnormality of neutrophil movement was an intrinsic property of the cells (Miller et al., 1971). This disorder has been known as the 'lazy leucocyte syndrome' (LLS). We describe a further case and report observations suggesting that the pathophysiological basis of LLS lies in an alteration in the structure or function of the microfilamentous protein of the granulocyte membrane.

\section{Methods}

Routine haematological investigations were carried out on a Coulter Counter Model S and by the methods of Dacie and Lewis (1975). Recruitment of neutrophils and monocytes to a site of inflammation was assessed by the 'skin window' technique of

Received for publication 24 August 1977
Rebuck and Crowley (1955), using a 12-mm diameter round coverslip, replaced at two- or four-hourly intervals for a period of 20 hours, over an abraded area on the volar aspect of the forearm. The cells adhering to the coverslips were stained with Romanowsky stain, examined by light microscopy, and counted. Mobilisation of neutrophils from marrow (Bishop et al., 1968; Cream, 1968) and marginal pools (Athens et al., 1961; Joyce et al., 1976) was studied after stimulation with hydrocortisone sodium succinate (100 mg intravenously) or adrenaline $(0.5 \mathrm{ml}$ of a $1 / 1000$ dilution intramuscularly) respectively. With hydrocortisone total and differential white cell counts were performed immediately before and hourly for six hours after administration. With adrenaline total and differential white cell counts were performed before and at 5,15 , 30,45 , and 60 minutes after administration. Random mobility of leucocytes was measured according to Bryant et al. (1966).

For in-vitro studies of neutrophil function neutrophil suspensions were prepared from heparinised blood by collecting white cell-rich plasma after sedimentation of red cells by adding a one-fifth volume of $6 \%$ dextran. After centrifugation of the white cell rich plasma the cell button was washed in Hanks's balanced salt solution (HBSS) and the cells were resuspended in HBSS at the required concentration. For experiments using vinblastine sulphate a final concentration of $5 \times 10^{-4} \mathrm{M}$ of the drug was used. Chemotaxis was studied by the methods of Boyden (1962), as modified by Clark and Kimball (1971), with a $3-\mu$ Millipore filter. Test and control 
cell suspensions were adjusted to provide $5 \times 10^{6}$ neutrophils $/ \mathrm{ml}$ in the final suspension placed in the upper chamber.

Autologous and normal sera were activated with $E$. coli endotoxin (Difco Laboratories, Detroit, Mich, USA) and used as attractants in the lower chamber. For studies with vinblastine (VBL) normal neutrophils were incubated at $22^{\circ} \mathrm{C}$ for 10 minutes with $5 \times 10^{-4} \mathrm{M}$ VBL before introduction to the Boyden chamber. The chambers were incubated in $5 \% \mathrm{CO}_{2}$ for three hours, when the filters were recovered, stained, and examined for cells that had passed through the membrane. Phagocytic capacity was examined according to El-Maalen and Fletcher (1976). Normal, patient, and vinblastine-treated cells (see above) were incubated with Candida albicans at concentrations of $1 \times 10^{6} / \mathrm{ml}$ cells and $5 \times 10^{6} / \mathrm{ml}$ organism in $\mathrm{HBSS}$ at $37^{\circ} \mathrm{C}$. Aliquots were removed at $3,5,10,15,20,30$, and 40 minutes, placed in cold saline, centrifuged, and examined. After staining, 800-1000 Candida were counted and the proportion phagocytosed was expressed as a percentage. Bactericidal capacity was assessed using Staphylococcus aureus 502A according to Quie et al. (1967). Mixtures of organism and neutrophils were incubated at $37^{\circ} \mathrm{C}$ for three hours. Samples were removed at 20,90 , and 180 minutes, diluted, and used in a pourplate of nutrient agar. Colony counts were performed after 48 hours at $37^{\circ} \mathrm{C}$. Vinblastine-treated cells were tested both before and after washing to remove vinblastine, and little difference was seen in the results.

Candidacidal capacity was determined by the method of Lehrer and Cline (1969) using patient, normal, and vinblastine-treated normal cells. Peroxidase staining was carried out according to Kaplow (1965) and nitroblue tetrazolium reduction was determined by the techniques of Park et al. (1968) and Park and Good (1970).

For scanning electron microscopy whole blood from the patient and a normal individual was layered over Hypaque-Ficoll and then centrifuged. The band of mononuclear cells was removed and the red cells and neutrophils were separated using dextran, as described above. Normal and patient neutrophils ( $>95 \%$ pure) were resuspended in HBSS at a concentration of $5 \times 10^{6} / \mathrm{ml}$. An aliquot of normal cells was treated with vinblastine for two hours at $37^{\circ} \mathrm{C}$. The cells were then fixed in $1 \%$ glutaraldehyde in phosphate buffered saline (PBS), as described by Fujita et al. (1971). After fixation overnight at $4^{\circ} \mathrm{C}$ the cells were washed in PBS and dehydrated in graded alcohols. The final suspension in $100 \%$ alcohol was gold plated and examined on a Cambridge Stereoscan electron microscope, Mark 2A (Kupari-Koby, 1976).
The assay for granulopoietic colony formation in culture was similar to that of Iscove et al. (1971). Bone marrow cells were suspended in methyl cellulose in alpha medium (Floro Laboratories) with $20 \%$ fetal calf serum in the presence or absence of $20 \%$ leucocyte conditioned medium (LCM). After 14 days in culture at $37^{\circ} \mathrm{C}$ in a high humidity, $7.5 \% \mathrm{CO}_{2}$ in air atmosphere granulocyte and macrophage colonies were counted. Two plates were counted for each group. In all experiments bone marrow cells were prepared as 'adherent' and 'non-adherent' (NA) fractions before culture (Senn et al., 1974).

\section{Case report}

The patient, a 40-year-old Caucasian woman, was referred for investigation of a neutropenia of at least 13 years' duration. She gave a history of upper respiratory tract infections; tonsillitis and repeated sore throats (before and after tonsillectomy at 18 years of age); recurrent bouts of sinusitis treated with antibiotics, antrostomy, and antrectomy; gingivitis; conjunctivitis; vaginitis; and pustular skin infections since childhood. Her two brothers and her two children did not suffer unduly from infections. Physical examination was unremarkable.

The total leucocyte count was $1.5 \times 10^{9} / 1(1500 /$ $\left.\mathrm{mm}^{3}\right)$ with $7 \%$ neutrophils $\left(0 \cdot 105 \times 10^{9} / 1\left(105 / \mathrm{mm}^{3}\right)\right)$. Absolute neutrophil counts subsequently were consistently less than $0.600 \times 10^{9} / 1\left(600 / \mathrm{mm}^{3}\right)$. The bone marrow was of normal cellularity with normal iron stores. Erythropoiesis was normoblastic. The myeloid:erythroid ratio was normal $(3: 1)$ and a differential count of 500 cells gave the following proportions of neutrophils: myelocytes $18 \%$, metamyelocytes $25.6 \%$, mature neutrophils and bands $11 \cdot 8 \%$. Megakaryocytes were unremarkable. Neutrophil morphology in blood films and in bone marrow was normal on light microscopy. Myeloperoxidase activity and reduction of nitroblue tetrazolium were normal.

Tests of immunological function gave the following results: Immunoelectrophoresis of patient's serum showed IgG $1476 \mathrm{mg} / \mathrm{dl}, \operatorname{IgA} 225 \mathrm{mg} / \mathrm{dl}$, IgM $105 \mathrm{mg} / \mathrm{dl}$ (all within normal range), and IgE 375 IU (normal range 10-300 IU). Stimulation of lymphocytes by phytohaemagglutinin, concanaval in $A$, and pokeweed mitogen produced normal levels of incorporation of tritiated thymidine.

Investigations of in-vitro proliferation of granulocytes showed normal granulocyte and monocyte colony formation in both number and morphology. Colony-stimulating activity (CSA) prepared from the patient's leucocytes was capable of stimulating colony formation (Table 1). 
Table 1 In-vitro colony formation by marrow cells

\begin{tabular}{llll}
\hline $\begin{array}{l}\text { Source of non- } \\
\text { adherent marrow } \\
\text { cells }\end{array}$ & $\begin{array}{l}\text { Source of } \\
\text { LCM }\end{array}$ & Colonies $(C F U-C)$ \\
\cline { 3 - 4 }$\left(1 \times 10^{5}\right)$ & & Granulocyte & Macrophage \\
\hline Normalt & - & 5 & \\
& Standard & 63 & 0 \\
Patient & Patient & 68 & 5 \\
& Standard & 2 & 4 \\
& Patient & 82 & 0 \\
& & & 5 \\
& & & 4
\end{tabular}

* Leucocyte conditioned medium prepared from leucocytes of a normal person (standard) or those of the patient.

tNormal marrow cells obtained from a person with mild iron deficiency.

\section{INVESTIGATIONS OF GRANULOCYTE} FUNCTION

The maximum number of neutrophils attracted to the Rebuck skin window was 351 on a $37.7 \mathrm{~mm}^{2}$ coverslip six hours after abrasion of the skin. Normal individuals from 4 to 20 hours after abrasion have $>1000$ neutrophils in the same area. The monocyte response was normal, $>1000$ monocytes being present 6 to 20 hours after abrasion.

Stimulation with adrenaline produced a maximum increment in the absolute neutrophil count of $0.765 \times 10^{9} / 1\left(765 / \mathrm{mm}^{3}\right) 60$ minutes after injection. At 5,15 , and 30 minutes increments in the neutrophil counts were $0.060 \times 10^{9} / 1$, nil, and $0.150 \times$ $10^{9} / 1\left(60 / \mathrm{mm}^{3}\right.$, nil, and $\left.150 / \mathrm{mm}^{3}\right)$ respectively. The normal range of maximal response in our laboratory is $0.300-4.20 \times 10^{9} / 1\left(300-4200 / \mathrm{mm}^{3}\right)$, mean $1.90 \times$ $10^{9} / 1\left(1900 / \mathrm{mm}^{3}\right)$, usually occurring within the first 30 minutes after giving adrenaline.

Stimulation with hydrocortisone produced a maximum increment in the absolute neutrophil count of $0.385 \times 10^{9} / 1\left(385 / \mathrm{mm}^{3}\right)$. The normal range in our laboratory is $0.600-5.40 \times 10^{9} / 1\left(600-5400 / \mathrm{mm}^{3}\right)$, mean $3.00 \times 10^{9} / 1\left(3000 / \mathrm{mm}^{3}\right)$.

Table 2 Comparison of the effects of the lazy leucocyte syndrome $(L L S)$ and treatment with $5 \times 10^{-4} M$ vinblastine on granulocyte function in vitro

\begin{tabular}{lcll}
\hline Function & Normal range & LLS & Vinblastine \\
\hline Random mobility & $0.4-1 \cdot 1 \mathrm{~mm}$ & $0.05 \mathrm{~mm}$ & $0.01 \mathrm{~mm}$ \\
Chemotactic index* & $14-178$ & $<1$ & $<1$ \\
Phagocytic capacity at: $\dagger$ & & & \\
3 min & $14-60$ & 16 & 19 \\
10 min & $21-66$ & 21 & 26 \\
15 min & $33-75$ & 31 & 39 \\
Bactericidal capacity $\ddagger$ & $0 \cdot 1-3 \cdot 8$ & $1 \cdot 3$ & $0 \cdot 3$ \\
Candidacidal activity $\S$ & $17-34$ & $4,7 \|$ & $2,9 \|$ \\
\hline
\end{tabular}

* Mean number of cells $10 /$ hpf migrating through Millipore filter.

$\dagger$ Per cent of particles ingested.

$\ddagger$ Per cent survival of organism.

§er cent killing.

\|Two separate estimations.
Table 2 shows the results of other investigations of neutrophil function and compares them with the results using normal granulocytes exposed to $5 \times$ $10^{-4} \mathrm{M}$ vinblastine (see below). Random mobility and directed migration (chemotactic index) were both reduced. The impairment of chemotactic capacity was intrinsic to the patient's neutrophils, since normal neutrophils migrated normally when the patient's serum was used to suspend them and to generate chemotactic attractant. Conversely, the patient's neutrophils showed impaired chemotactic mobility when normal serum was used in the experimental system. The phagocytic capacity of the patient's neutrophils at all times of incubation was at the lower limit of normal. Bactericidal capacity was within normal limits but candidacidal activity was reduced. Nitroblue tetrazolium reduction and myeloperoxidase activity by cytochemical assessment were both normal. Serum lysozyme concentration was 1.0 and $2.1 \mu \mathrm{g} / \mathrm{ml}$ on two occasions (normal $<7 \mu \mathrm{g} / \mathrm{ml})$.

\section{SCANNING ELECTRON MICROSCOPY}

The appearances of normal granulocytes prepared by Hypaque-Ficoll gradient separation followed by resuspension in Hanks's buffered salt solution (HBSS) are shown in Figs 1 and 2. The normal spheroidal form shows fine ruffles and occasional small, knob-like projections (Fig. 1). Figure 2 shows an amoeboid form with large filmy ruffles as well as finer small ruffles. Neutrophils from the patient are shown in Figs 3 and 4.

Figure 3 shows a spherical form in which the surface configurations are coarser than the normal. Knob-like projections predominate over fine ruffles and some of the knob-like projections are of considerable size. In Fig. 4 an amoeboid form is seen which shows predominantly small, knob-like projections with substantial featureless areas between and loss of fine and large filmy ruffles compared with the normal amoeboid form. The other cell shows coarse, large ruffles.

\section{EFFECTS OF EXPOSURE TO VINBLASTINE} In-vitro exposure of normal neutrophils to $5 \times 10^{-4} \mathrm{M}$ vinblastine produces functional alterations in neutrophil behaviour similar to those produced by the defect leading to LLS. In Table 2 the results of tests of granulocyte function after exposure to vinblastine are compared with those using LLS and normal neutrophils. Random and directed migration are both seriously impaired, and phagocytic capacity, although within the normal range, is at the lower limit of normal. Bactericidal capacity is normal and killing of Candida albicans is impaired. 


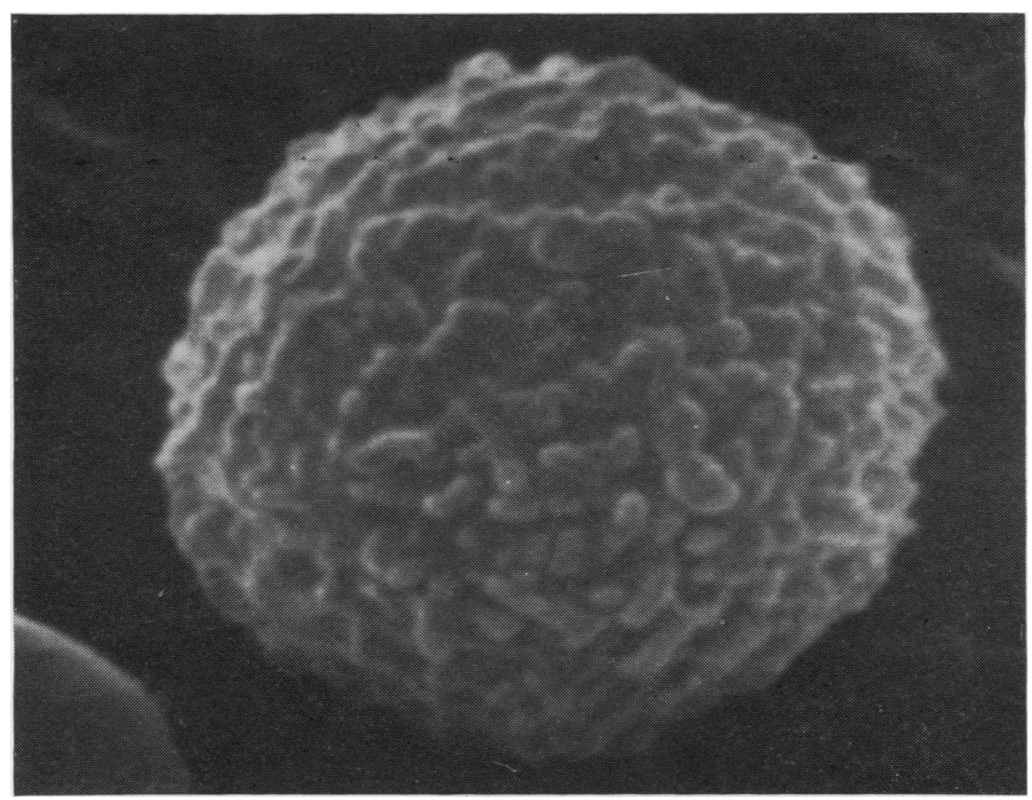

Fig. 1 Normal neutrophil, spherical form, showing regular small fine ruffles and occasional poorly defined small, knob-like projections with little space between surface elevations. $(\times 11400)$

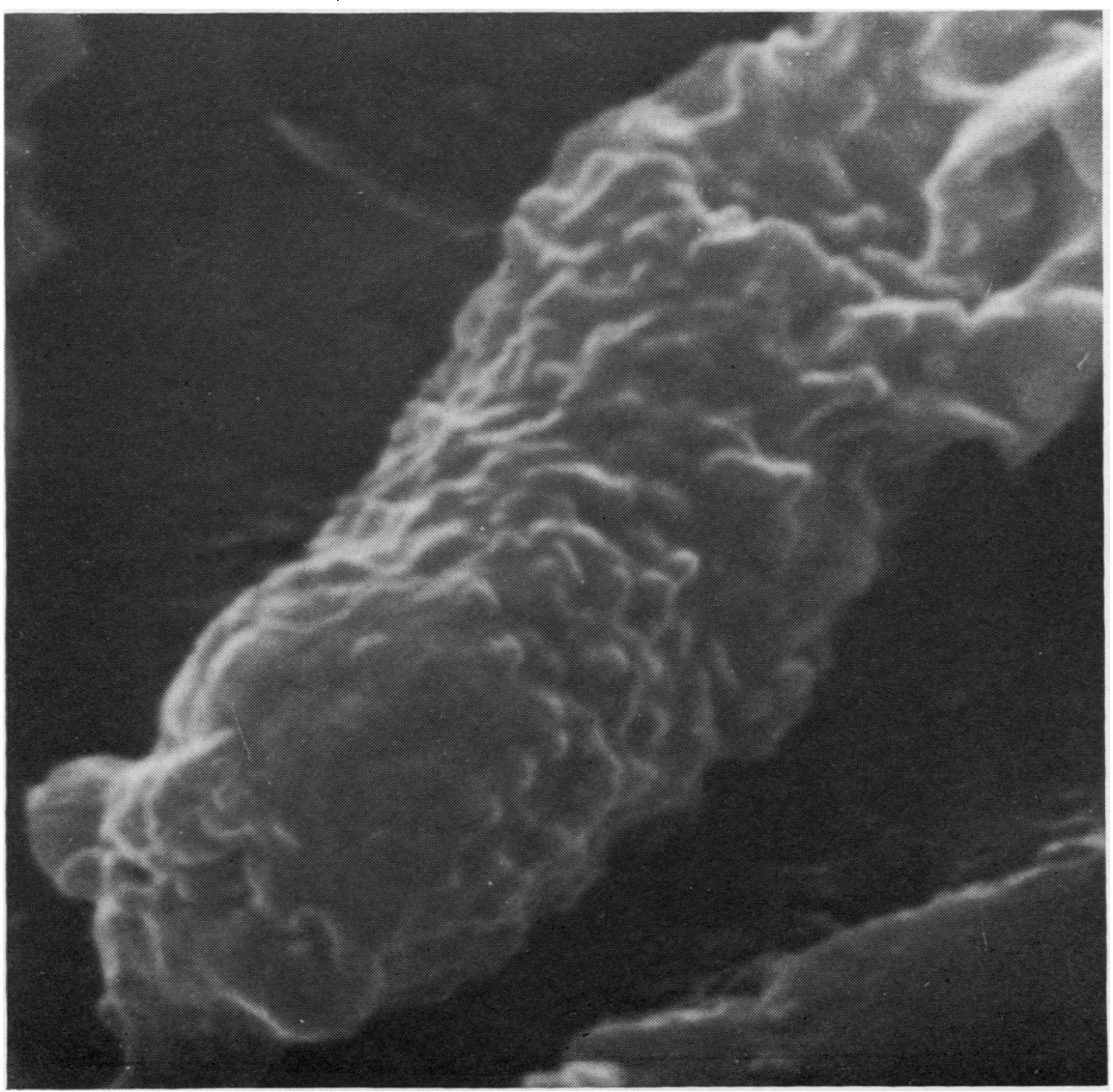

Fig. 2 Normal neutrophil, amoeboid form, showing numerous small, fine ruffles and with larger filmy ruffles at the leading end. $(\times 11400)$ 


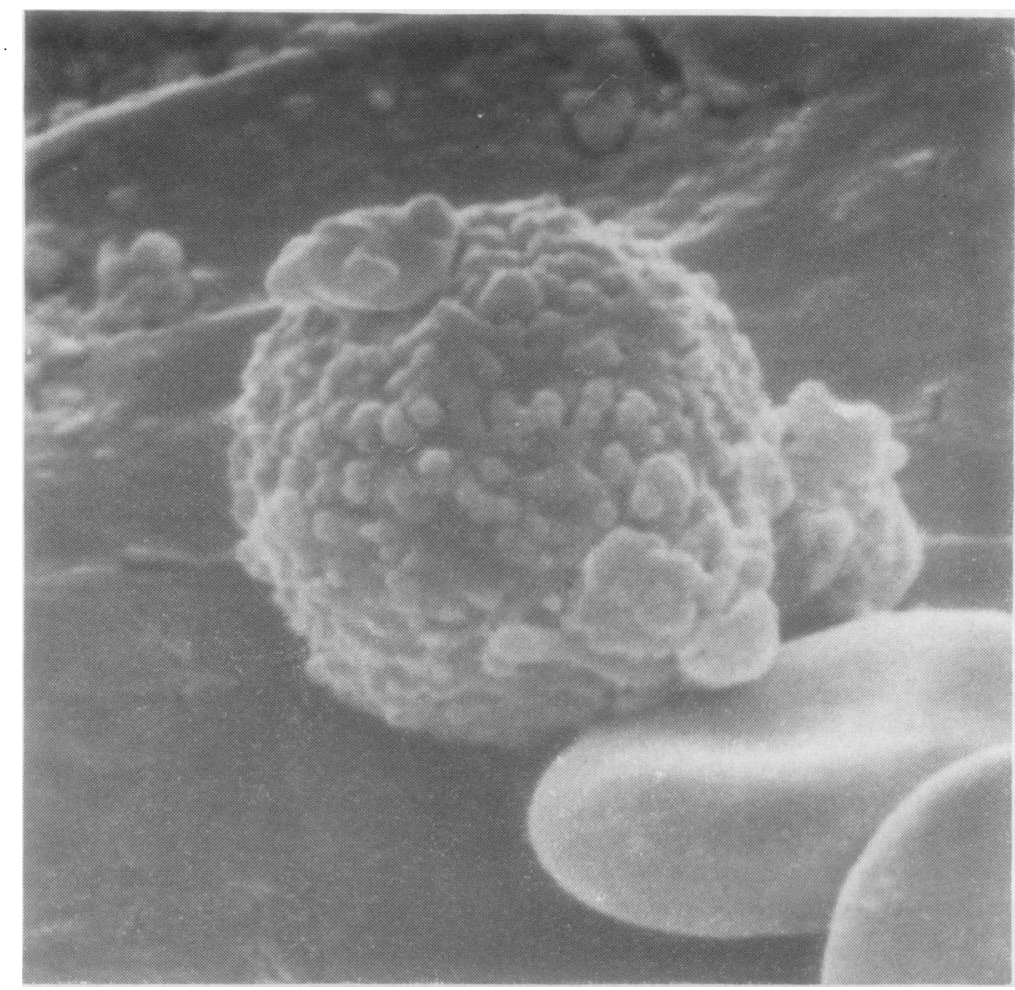

Fig. 3 Neutrophil, spherical form, in the lazy leucocyte syndrome showing numerous small. knob-like projections and multiple, large knob-like projections. $(\times 11400)$

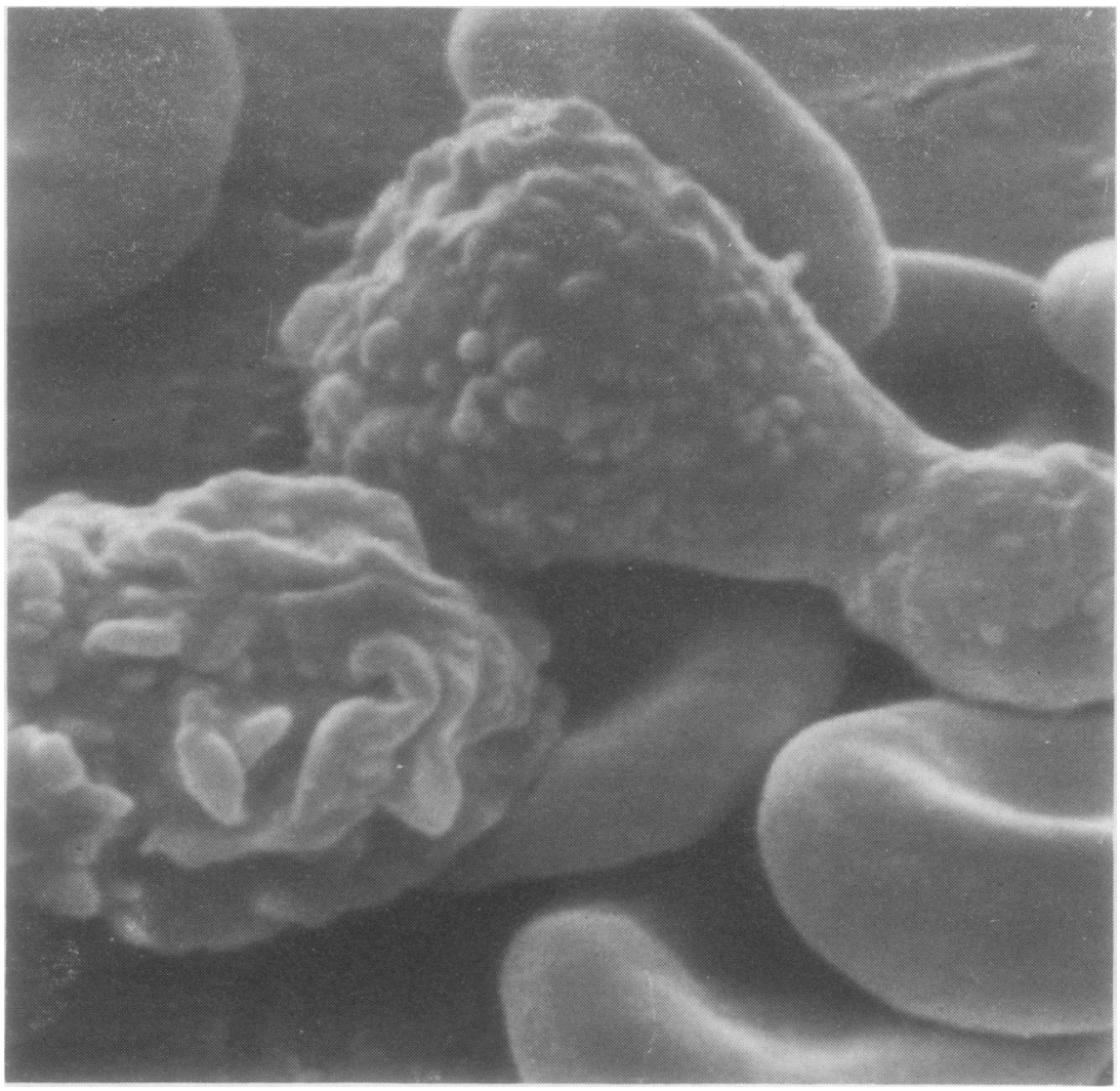

Fig. 4 Two neutrophils in the lazy leucocyte syndrome. The upper, an amoeboid form, shows loss of both fine and large filmy ruffles with, instead, small, irregularly distributed, knob-like projections and occasional poorly developed ruffles with extensive featureless areas between. The lower shows coarsening of the ruffled pattern. $(\times 11400)$ 


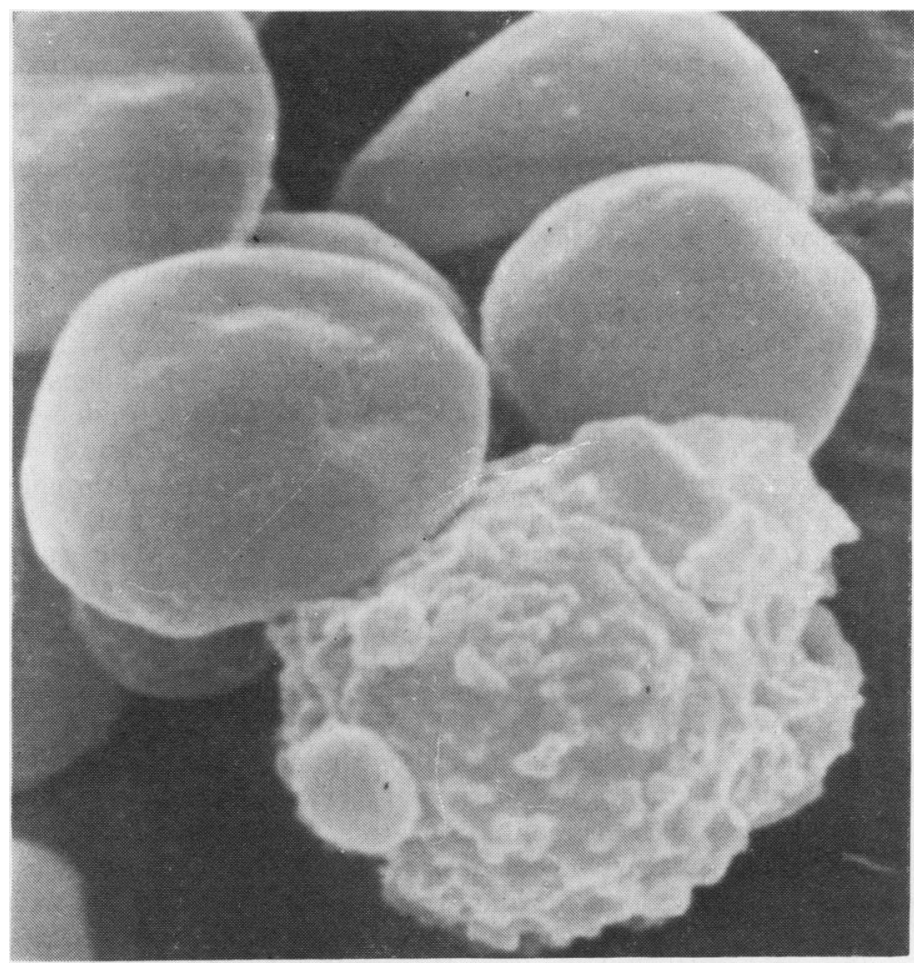

Fig. 5 Normal neutrophil, spherical form, treated with vinblastine showing coarsening and irregularity of ruffles and large, knob-like projections. $(\times 11400)$

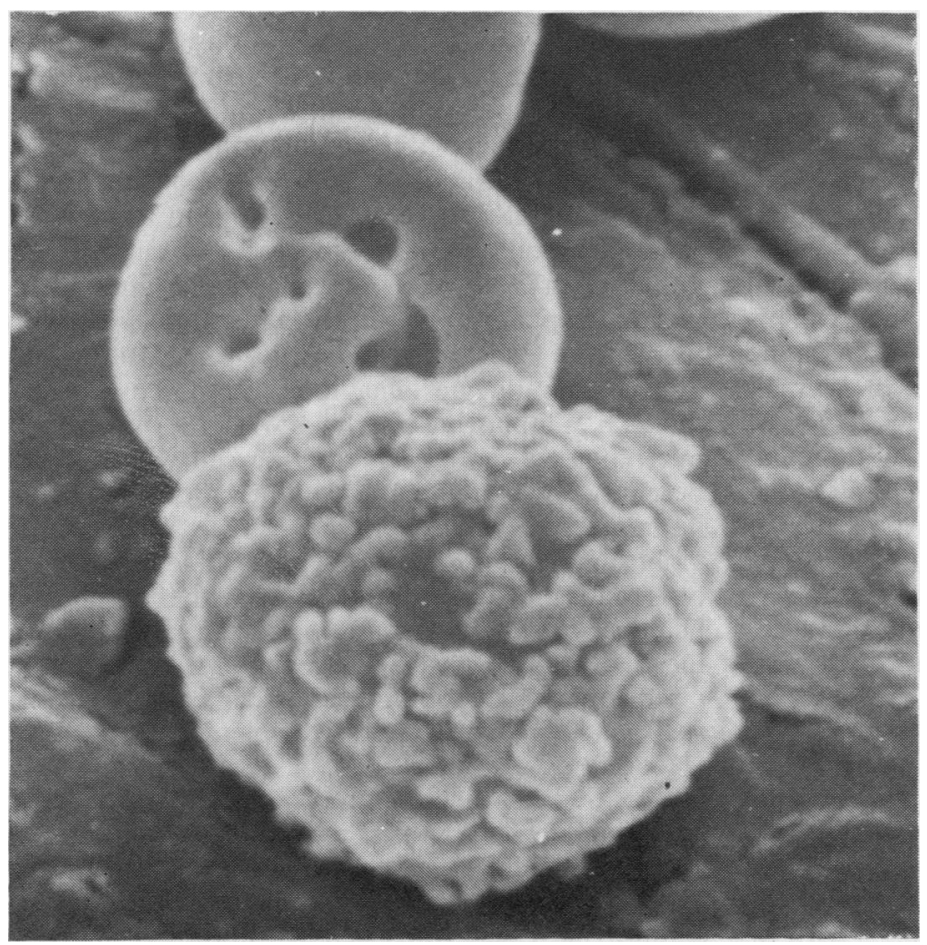

Fig. 6 Neutrophil, spherical form, in the lazy leucocyte syndrome treated with vinblastine, shows no obvious differences from the untreated neutrophils from the same patient (Fig. 3). Other cells showing large knob-like projections could also be found in the same preparation. Red cell showing vinblastine effects is also present. (×11 400) 
On scanning electron microscopy of vinblastinetreated normal neutrophils the fine ruffled pattern is disorganised. The ruffles are coarse and more irregular than normal and large, knob-like projections are seen (Fig. 5). The amoeboid forms show loss of the ruffled pattern with, instead, irregular distribution of short, rather coarse ruffles and small, knob-like projections with large featureless areas of cell surface, similar to the appearances seen in the upper cell in Fig. 4. Treatment with vinblastine has comparatively little effect on the surface configuration of neutrophils from the patient with LLS (Fig. 6: compare with Fig. 3).

\section{Discussion}

The abnormalities of granulocyte behaviour observed in our patient are very similar to those described by Miller et al. (1971) in the lazy leucocyte syndrome in two children and by Constantopoulos et al. (1975) in another child. All cases described hitherto have been in children, and Miller et al. (1971) have emphasised the mildness of the condition. Our patient, now an adult, has led an active life little inhibited by her disorder of neutrophil function.

Patients with LLS present with severe peripheral neutropenia associated with normal granulocyte maturation and morphology on simple bone marrow examination. Further, we have shown normal invitro proliferative capacity of LLS granulocyte precursors-colony-forming units in culture (CFUC) are present in normal numbers and production of colony-stimulating activity (CSA) is also normal. These results are in keeping with previous reports in neutropenia from various causes (Greenberg and Schrier, 1973; Senn et al., 1974).

The circulating pool of neutrophils is reduced in size and the number of marginated pool neutrophils released into the circulating pool by adrenaline (Athens et al., 1961) is in the low range of normal. Stimulation of release of the marrow reserves of neutrophils by steroids (Bishop et al., 1968; Cream, 1968) produces only a small increase in the peripheral neutrophil count in spite of the presence of ample mature-looking neutrophils in the bone marrow and normal in-vitro proliferative capacity. Migration of neutrophils to skin windows is defective, but that of monocytes appears to be at least as good as in normal individuals.

Investigations of granulocyte function in vitro demonstrate that the abnormalities in migration are intrinsic to the granulocyte. Migration of granulocytes through a Millipore filter in response to a chemotactic stimulus is deficient. The cellular response of the patient's granulocytes is poor whether normal control or patient's serum is used to suspend the white cells and produce the chemotactic stimulus. Conversely, the patient's serum supports a normal chemotactic response by normal granulocytes.

In spite of the deficiencies of neutrophil mobility, whether random or directed, the ability to ingest particles and kill micro-organisms is not seriously compromised. Phagocytic capacity is at the lower limits of the normal range, bacterial killing is normal, and fungal killing moderately impaired with the in-vitro techniques we employed. Alterations in proportions of particles or organisms to granulocytes might have produced more sensitive measures of these functions but insufficient patient neutrophils were available for more extensive experiments.

In their original description of LLS, Miller et al. (1971) postulated that the disorder of granulocyte function involved the cell membrane. This, combined with the observations of Lichtman and his colleagues (Lichtman, 1970; Lichtman and Weed, 1972; Giordano et al., 1973) that cell rigidity might be a determinant in controlling release of granulocytes from bone marrow when they reach the later stages of maturation, lead us to postulate that the LLS may be a disorder of the polymorphonuclear membrane leading to altered deformability, perhaps analogous to hereditary spherocytosis of the red cell, where there appears to be disordered function of the microfilamentous protein of the cell membrane (Valentine, 1977). Such undue rigidity could explain failure of egress from the bone marrow, failure of migration to the skin window, and failure of the cellular aspects of directed mobility (chemotaxis) through a Millipore filter. Altered membrane function might also be expected to lead to impaired random mobility and would be consistent with the impaired or below-average phagocytic and microbicidal capacity. To investigate this possibility further the effect of vinblastine $\left(5 \times 10^{-4} \mathrm{M}\right)$ on normal granulocyte behaviour was compared with the LLS. Vinblastine interferes with the function of microfilamentous proteins of the cell wall and has been used with the same concentration on normal human erythrocytes in vitro. Such exposure to vinblastine induces spherocytic changes in the red cells and reproduces very closely the findings in hereditary spherocytosis-increased cell rigidity, increased osmotic fragility, increased membrane permeability to sodium, and decreased red cell survival in vivo with excessive splenic sequestration (Jacob et al., 1972).

Colchicine, which also reproduces the changes of hereditary spherocytosis in red cells on in-vitro exposure, inhibits directed mobility of polymorphs (Caner, 1965) and interferes with amoeboid motility (Malawista, 1965). More detailed studies showed that 
vinblastine and colchicine had similar effects on polymorphonuclear function, both producing inhibition of oxygen consumption and $\mathrm{CO}_{2}$ production, lysozomal degranulation, and digestive vacuole formation. However, phagocytosis itself and the capacity to kill Staph. aureus are not obviously impaired (Malawista, 1971). Edelson and Fudenberg (1973) observed impaired chemotactic responsiveness but no inhibition of random mobility after exposure to $0.2 \times 10^{-4} \mathrm{M}$ vinblastine sulphate. The results presented in Table 2 show that exposure of neutrophils to vinblastine reproduces closely the functional abnormalities seen in LLS.

The appearances of the neutrophils in LLS on scanning electron microscopy are abnormal. The rather fine and filmy ruffles normally seen are replaced by coarser more irregular patterns, and knob-like projections, occasionally seen in the normal, predominate over fine ruffles. In amoeboid forms coarsening of ruffles and increase in the featureless areas between ruffles are present. This is interpreted as further evidence of membrane abnormality in LLS. When normal neutrophils are exposed to vinblastine there is disorganisation and irregularity of the ruffled pattern and the appearance of coarse, knob-like projections similar to those seen in LLS. Normal amoeboid forms lose their large filmy and fine ruffles, showing instead short irregular ruffles and small, knob-like projections similar to those seen in LLS.

Similar changes have been described in normal neutrophils exposed to colchicine and vinblastine (Lichtman et al., 1976). The resemblance of changes in LLS neutrophils to those produced by vinblastine and colchicine in this study and by others (Lichtman et al., 1976) would be in keeping with the suggestion that both circumstances affect the function of the microtubular proteins of the cell membrane. The observation that vinblastine produces no obvious additional modification of the appearances of LLS neutrophils suggests that the cellular lesion of the LLS neutrophils and that produced by vinblastine affect the neutrophil membrane in the same or a similar fashion.

The presence of normal numbers of monocytes in the skin windows suggests that the abnormality seen in the neutrophils is not present or is not expressed, at least not fully, in monocytes in spite of their close ancestral relationship (Cline, 1975; Stanley et al., 1975).

LLS is a clinically mild disorder which presents as neutropenia with undue liability to infection, in the presence of a normal bone marrow morphology, and characterised by defects in neutrophil mobility. The abnormal behaviour of the neutrophils in LLS may be explained by excessive rigidity. The experiments described here lend support to the hypothesis that the pathogenesis of LLS may be in dysfunction of the microtubular proteins of the neutrophil membrane.

We thank Dr Marshall A. Lichtman for helpful advice in the investigation of this patient and Mrs Helen Kupari-Koby for assistance in preparing the scanning electron micrographs.

\section{References}

Athens, J. W., Raab, S. O., Haab, O. P., Mauer, A. M., Ashenbrucker, H., Cartwright, G. E., and Wintrobe, M. M. (1961). Leukokinetic studies III. The normal distribution of granulocytes in the blood of normal subjects. Journal of Clinical Investigation, 40, 159-164.

Baehner, R. L. (1974). Molecular basis for functional disorders of phagocytes. Journal of Pediatrics, 84, 317327.

Bishop, C. R., Athens, J. W., Boggs, D. R., Warner, H. R., Cartwright, G. E., and Wintrobe, M. M. (1968). Leukokinetic studies XIII. A non-steady-state kinetic evaluation of the mechanism of cortisone-induced granulocytosis. Journal of Clinical Investigation, 47, 249260.

Boyden, S. (1962). The chemotactic effect of mixtures of antibody and antigen on polymorphonuclear leucocytes. Journal of Experimental Medicine, 115, 453-466.

Bryant, R. E., DesPrez, R. M., VanWay, M. H., and Rogers, D. E. (1966). Studies on human leukocyte motility. Journal of Experimental Medicine, 124, 483499.

Caner, J. E. Z. (1965). Colchicine inhibition of chemotaxis. Arthritis and Rheumatism, 8, 757-764.

Clark, R. A., and Kimball, H. R. (1971). Defective granulocyte chemotaxis in the Chediak-Higashi syndrome. Journal of Clinical Investigation, 50, 2645-2652.

Cline, M. J. (1975). The White Cell. Harvard University Press, Cambridge, Massachusetts, and London.

Constantopoulos, A., Karpathios, T., Nicolaidou, P., Maounis, F., and Matsaniotis, N. (1975). Lazyleukocyte syndrome. A case report. Journal of Pediatrics, 87, 945-946.

Cream, J. J. (1968). Prednisolone-induced granulocytosis. British Journal of Haematology, 15, 259-267.

Dacie, J. V., and Lewis, S. M. (1975). Practical Haematology, 5th edition. Churchill Livingstone, Edinburgh.

Edelson, P. J., and Fudenberg, H. H. (1973). Effect of vinblastine on the chemotactic responsiveness of normal human neutrophils. Infection and Immunity, 8, 127129.

El-Maalen, H., and Fletcher, J. (1976). Defective neutrophil function in chronic granulocytic leukaemia. British Journal of Haematology, 34, 95-103.

Fujita, T., Tokunaga, J., and Inoue, H. (1971). Atlas of Scanning Electron Microscopy in Medicine. Elsevier, Amsterdam and New York.

Gallin, J. I., and Wolff, S. M. (1975). Leucocyte chemotaxis: physiological considerations and abnormalities. Clinics in Haematology, 4, 567-607. 
Giordano, G. F., Lichtman, M. A., and Mayle, E. (1973). Marrow cells egress. The central interaction of barrier pore size and cell maturation. Journal of Clinical Investigation, 52, 1154-1164.

Greenberg, P. L., and Schrier, S. L. (1973). Granulopoiesis in neutropenic disorders. Blood, 41, 753-769.

Iscove, N. N., Senn, J. S., Till, J. E., and McCulloch, E. A. (1971). Colony formation by normal and leukemic human marrow cells in culture: effect of conditioned medium from human leukocytes. Blood, 37, 1-5.

Jacob, H. S., Amsden, T., and White, J. (1972). Membrane microfilaments of erythrocytes: Alterations in intact cells reproduces the hereditary spherocytosis syndrome. Proceedings of the National Academy of Sciences of the United States of America, 69, 471-474.

Joyce, R. A., Boggs, D. R., Hasiba, U., and Srodes, C. H. (1976). Marginal neutrophil pool size in normal subjects and neutropenic patients as measured by epinephrine infusion. Journal of Laboratory and Clinical Medicine, 88, 614-620.

Kaplow, L. S. (1965). Simplified myeloperoxidase stain using benzidine dihydrochloride. Blood, 26, 215-219.

Kupari-Koby, H. (1976). A comparison of blood cells by the light microscope and scanning electron microscope. Canadian Journal of Medical Technology, 38, 33-49.

Lehrer, R. 1., and Cline, M. J. (1969). Interaction of Candida albicans with human leucocytes and serum. Journal of Bacteriology, 98, 996-1004.

Lichtman, M. A. (1970). Cellular deformability during maturation of the myeloblast. Possible role in marrow egress. New England Journal of Medicine, 283, 943-948.

Lichtman, M. A., Santillo, P. A., Kerney, E. A., Roberts, G. W., and Weed, R. I. (1976). The shape and surface morphology of human leucocytes in vitro: effect of temperature, metabolic inhibitors and agents that influence membrane structure. Blood Cells, 2, 507-531.

Lichtman, M. A., and Weed, R. I. (1972). Alteration of the cell periphery during granulocyte maturation: $\frac{0}{\vec{J}}$ relationship to cell function. Blood, 39, 301-316.

Malawista, S. E. (1965). The action of colchicine in acute $\Rightarrow$ gout. Arthritis and Rheumatism, 8, 752-756.

Malawista, S. E. (1971). Vinblastine: Colchicine-like $\bar{C}$ effects on human blood leucocytes during phagocytosis. Blood, 37, 519-529.

Miller, M. E. (1975). Pathology of chemotaxis and random mobility. Seminars in Hematology, 12, 59-82.

Miller, M. E., Oski, F. A., and Harris, M. B. (1971). Lazy-ळ leucocyte syndrome. A new disorder of neutrophil $\vec{D}$ function. Lancet, 1, 665-669.

Park, B. H., Fikrig, S. M., and Smithwick, E. M. (1968). $\vec{\omega}$ Infection and nitro-blue-tetrazolium reduction by neutrophils; a diagnostic aid. Lancet, 2, 532-534.

Park, B. W., and Good, R. A. (1970). N.B.T. test stimulated (Letter). Lancet, 2, 616-617.

Quie, P. G. (1975). Pathology of the bactericidal power of neutrophils. Seminars in Hematology, 12, 143-160.

Quie, P. G., White, J. G., Holmes, B., and Good, R. A. (1967). In vitro bacteriacidal capacity of human poly- 음 morphnuclear leucocytes: diminished activity in $\overrightarrow{-}$ chronic granulomatous disease of childhood. Journal of $\frac{D}{0}$ Clinical Investigation, 46, 668-679.

Rebuck, J. W., and Crowley, J. H. (1955). A method of $\vec{\theta}$ studying leucocytic functions in vivo. Annals of the New York Academy of Sciences, 59, 757-805.

Senn, J. S., Messner, H. A., and Stanley, E. R. (1974). Analysis of interacting cell populations in cultures of marrow from patients with neutropenia. Blood, 44, 3340.

Stanley, E. R., Hansen, G., Woodcock, J., and Metcalf, D. (1975). Colony stimulating factor and the regulation of granulopoiesis and macrophage production. Federation $\overline{\mathrm{O}}$ Proceedings, 34, 2272-2278.

Valentine, W. N. (1977). The molecular lesion of hereditary spherocytosis (HS): A continuing enigma. Blood, 49, 241-245. 